\title{
Acute Perineal Inflammation with Extensive Necrosis: A Devastating Result of Triamcinolone Acetonide Injection for Penile Augmentation
}

\author{
Woong Gyu Na, Hyoseob Lim, Sung Won Jung, Sung Hoon Koh \\ Department of Plastic and Reconstructive Surgery, Hallym University Sacred Heart Hospital, Hallym University Medical Center, Anyang, Korea
}

\begin{abstract}
Injection of foreign materials for genital augmentation which may induce local infection, ulceration or systemic lipogranuloma embolization resulting in sudden death have been performed in contaminated environment by non-medical practitioners. We present A 36-year-old man who had been given triamcinolone acetonide ointment injection on scrotum and penile shaft followed by skin and soft tissue necrosis. Even though eschar debridement and 10 days of copious irrigation, betadine-soaked dressing and intravenous antibiotics injections were performed, the soft tissue necrosis had been more progressed. Five weeks of daily irrigation and betadine soaking dressing and 3 times of more aggressive debridements under general anesthesia resulted in extensive defect exposing penis, both testes, suprapubic area and both inguinal areas. Bilateral pudendal thigh flaps were done to cover the scrotal defect and remnant area was covered by split-thickness skin graft. On postoperative 1 day, minor congestion was found on left flap tip area which was salvaged with $4 \%$ lidocaine topical but resulted in small-sized eschar formation. Additional debridement and closure was performed without tension. Other areas survived without skin loss or dehiscence.
\end{abstract}

Keywords: Inflammation, Triamcinolone acetonide, Perineum

\section{Introduction}

Since the first case of mineral oil injection in the penis was reported in 1899, foreign materials such as paraffin oils, mineral oils, petroleum jelly, and silicone have been directly injected in the penis for granuloma formation to enlarge its size by nonmedical practitioners [1-3]. Injections of other various substances, including metallic mercury and stone, have also been reported [3]. Such injections may induce a local infection, ulceration, or systemic lipogranuloma embolization, resulting in sudden death $[1,4]$. Herein, we describe the case of 36-year-old man who had triamcinolone acetonide (TA) ointment injected into his penis for augmentation. This very rare case involved extensive skin and soft tissue necrosis on the penis, scrotum, suprapubic area, and both inguinal regions.

\section{Case}

A 36-year-old man was admitted to our urology department with escharic tissue on the penile shaft just below the glans and scrotum (Fig. 1). His friend had injected Oramedy ointment (Dongkook Pharmaceutical, Seoul, Korea) into the penile shaft to enlarge its circumference. An initial debridement of the eschar was performed, revealing undemarcated and inflamed soft tissue (Fig. 2). Copious irrigations with repetitive debridements and betadine-soaked dressings were performed for 10 days.

\section{Case Report}

Received: May 27, 2017

Revised: August 7, 2017

Accepted: August 18, 2017

\section{Corresponding author:}

Hyoseob Lim, M.D., Ph.D

Department of Plastic and Reconstructive Surgery, Hallym University Sacred Heart Hospital, Hallym University Medical Center, 22 Gwanpyeong-ro 170beon-gil, Dongan-gu, Anyang 14068, Korea

Tel: +82-31-380-3781

Fax: +82-31-380-5980

E-mail: hyoseob.lim@gmail.com

No potential conflict of interest relevant to this article was reported.

This is an Open Access article distributed under the terms of the Creative Commons Attribution Non-Commercial license (http:/lcreativecommons.orglicenses/by-nc/4.C which permits unrestricted non-commercial use, distribution, and reproduction in any medium, provided the original work is properly cited.

(c) 2017 Korean Wound Management Society 
Na WG et al.

Penile inflammation after steroid injection

JWMR

Serologic tests for several needlestick injury associated disease such as hepatitis B and $\mathrm{C}$, human immunodeficiency virus were conducted and negative results were reported. Intravenous injections of vancomycin and piperacillin/tazobactam were administrated for infection control. However, the soft tissue necrosis became more progressed, with turbid pus formation (Fig. 3). The adjacent suprapubic area and both inguinal areas became inflamed with skin redness. Aggressive debridements extending into the adjacent areas were performed 3 times under general anesthesia. During the first aggressive debridement, the surrounding skin margin and inner necrotic tissue were debrided (preserving both spermatic cords), and the defect was found to extend into the suprapubic area. During second and third aggressive debridements, the inner inflamed soft tissue under both inguinal areas was progressively debrided and the tunica vaginalis was opened on both sides. The infection on the penis, scrotum, suprapubic area, and both inguinal areas was finally controlled, 5 weeks after the initial debridement (Fig. 4).

Reconstruction of this complex structure was performed using multiple methods. The penile shaft and suprapubic area were covered by a split-thickness skin graft. To cover the scrotal defect, reconstruction with bilateral pudendal thigh flaps was performed (Fig. 5). Both superficial perineal arteries were preoperatively identified using a hand-held Doppler. The flaps were elevated at the subfascial plane, and the do-

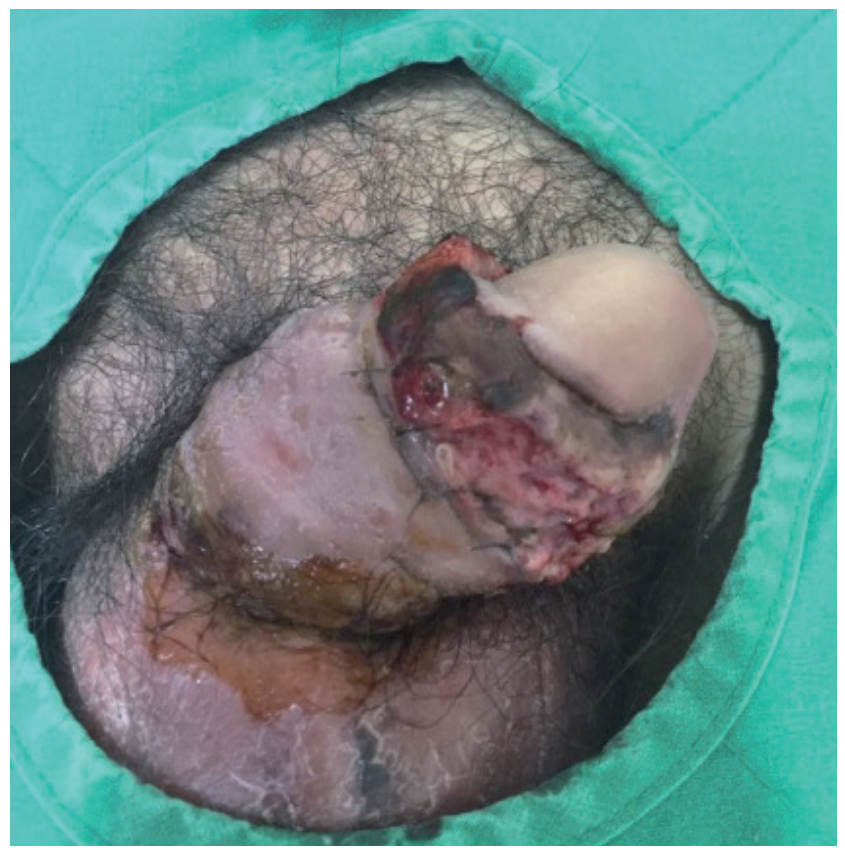

Fig. 1. Initial photograph at admission. nor sites were closed primarily. On postoperative day 1, minor distal flap congestion was observed in the left flap tip area, which was salvaged using $4 \%$ lidocaine topically, resulting in a small eschar formation (Fig. 6). Additional eschar debridement revealed partial-thickness skin loss and a primary clo-

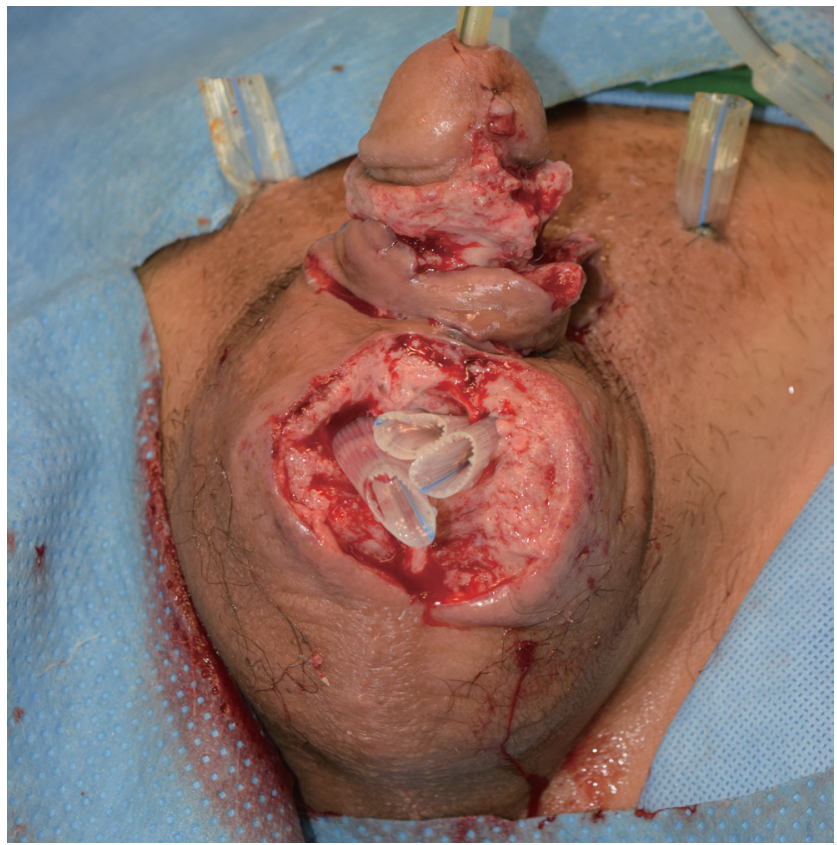

Fig. 2. Initial debridement photograph showing perineal inflammation.

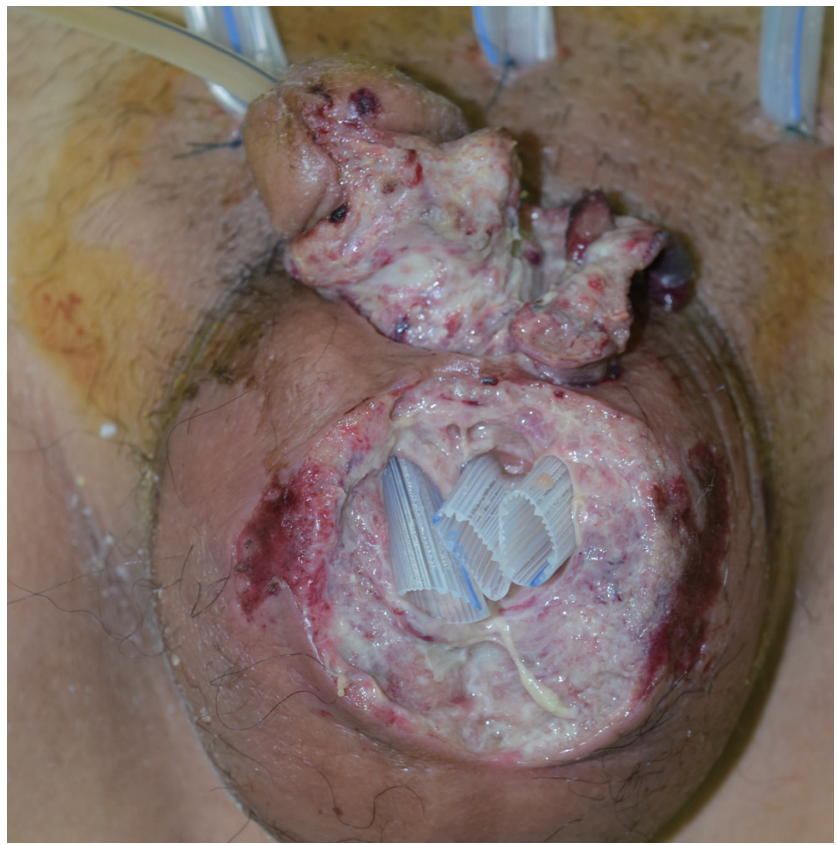

Fig. 3. Photograph after 10 days of daily irrigation. 
Na WG et al.

Penile inflammation after steroid injection

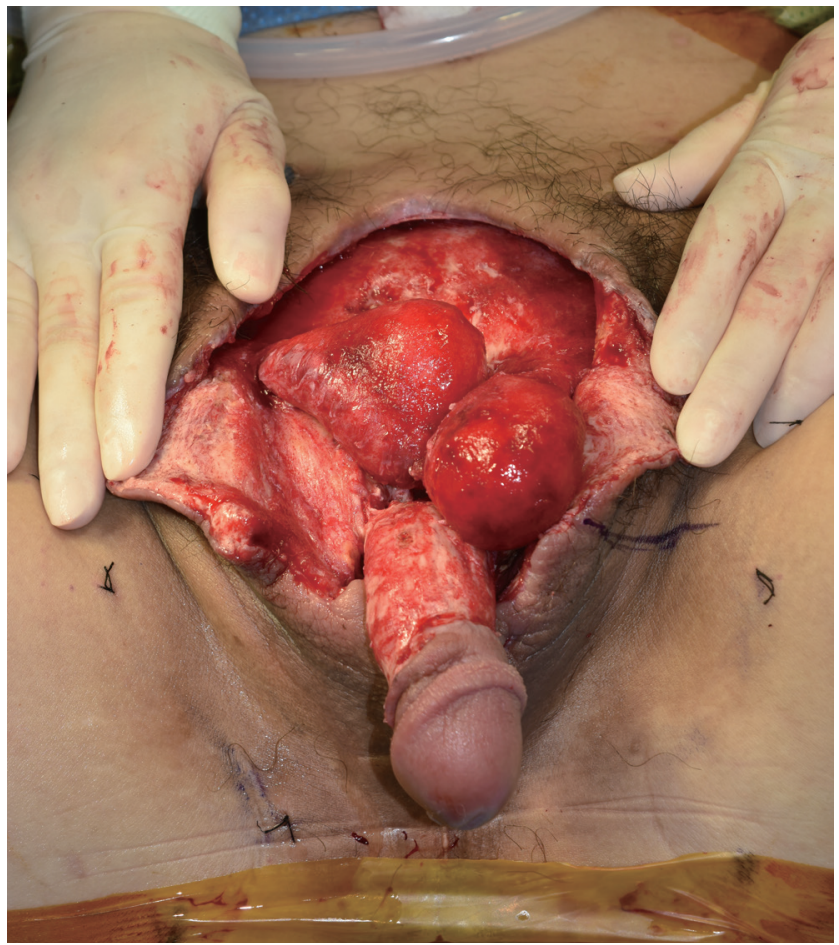

Fig. 4. Photograph after 5 weeks of daily irrigation.

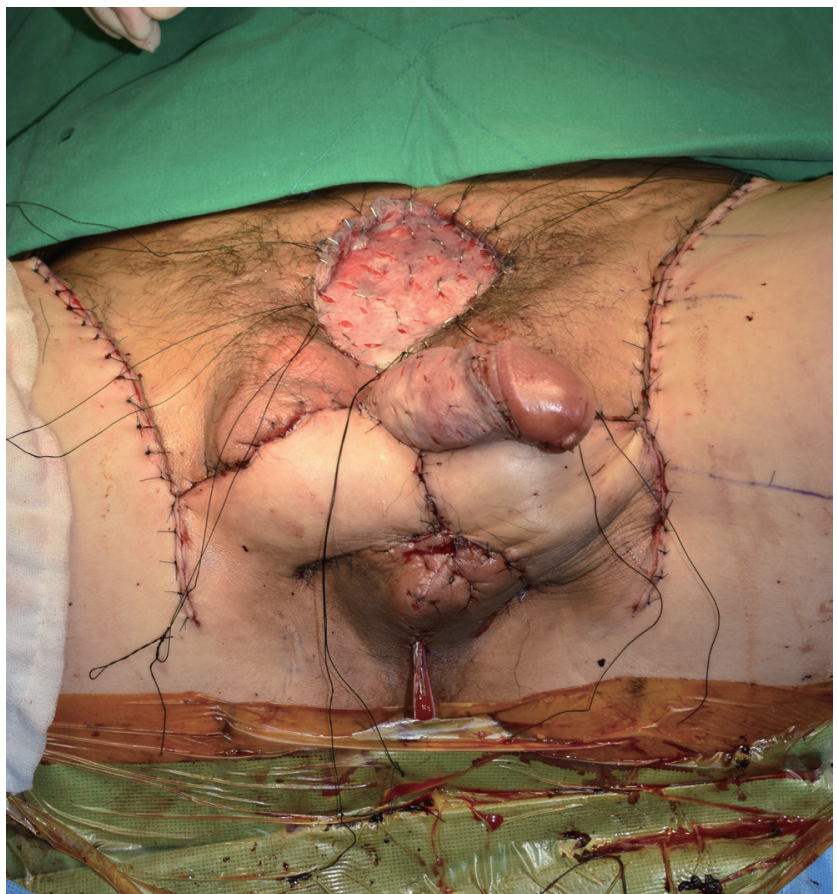

Fig. 5. Intraoperative photograph.

sure was performed without tension (Fig. 7). The patient gradually ambulated in a standing position at 1 week postoperative and sat on a toilet at 2 weeks postoperative.

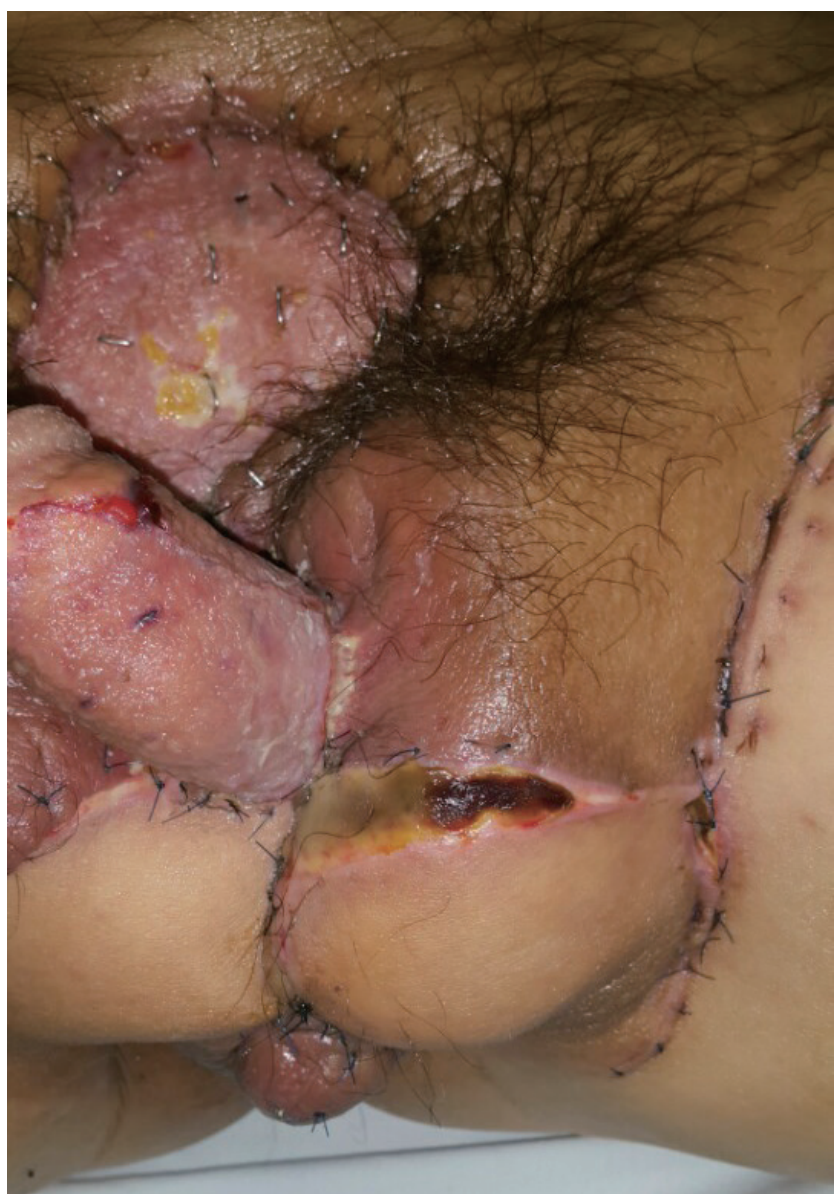

Fig. 6. Postoperative 18 days photograph.

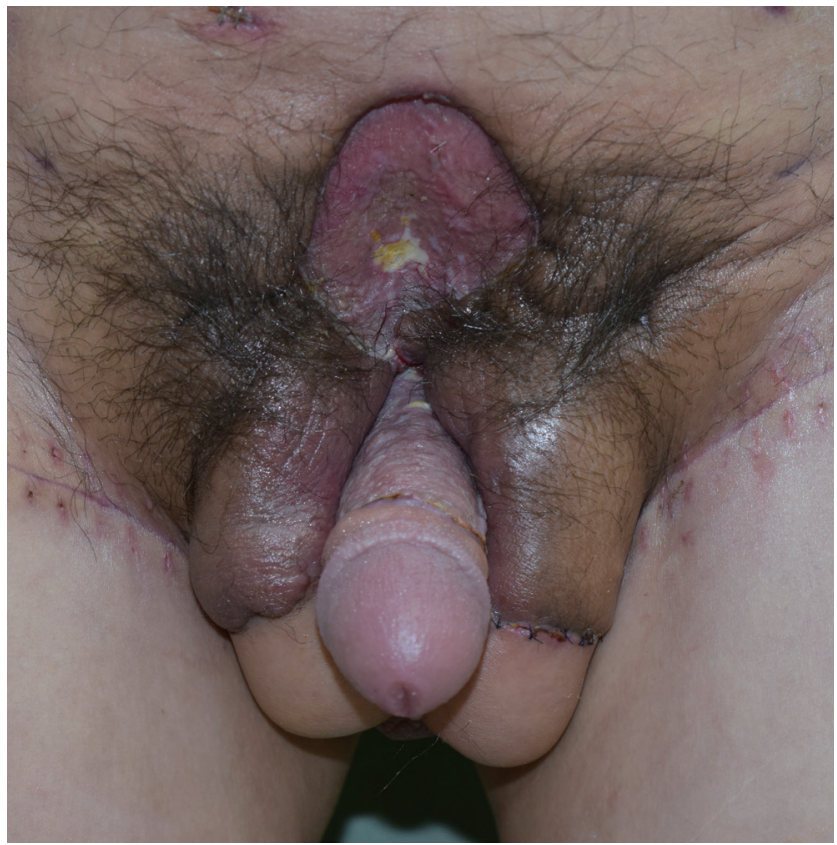

Fig. 7. Postoperative 24 days photograph. 


\section{Discussion}

In the present case, Oramedy ointment injected into the perineal area resulted in widespread acute inflammation, not only affecting the genital area, but also affecting the adjacent suprapubic and both inguinal areas. Oramedy ointment is mainly used to control intraoral ulcers; $1 \mathrm{~g}$ contains $1 \mathrm{mg}$ of TA and other emollient, protectant agents. TA reduces pain and inflammation and is an intermediate-acting glucocorticoid used for dermatoses, allergic rhinitis, and other oral inflammations, including aphthous stomatitis [5]. In addition, TA injections have been reported to reduce the size of rheumatoid nodules in patients with rheumatoid arthritis and are widely used in tenosynovitis [6,7]. The injection of TA in the perineal area is rare; its use other than that as an ointment for oral wounds has not been reported.

Despite daily dressings with irrigation and intravenous antibiotics, the local inflammatory changes in the penis and scrotal area were not controlled and progressed to widespread perineal inflammation accompanying skin and soft tissue necrosis. This devastating result could be caused by TA itself or by the other additive materials in the ointment. Complications involving inflammation or tissue necrosis after a corticosteroid injection have been reported [7]. Necrotizing fasciitis has occurred after an injection of TA into the greater trochanteric bursa and trigger finger [6]. Furthermore, epidural injections of TA can induce discitis or osteomyelitis [6]. Wide digital necrosis, potentially resulting from a direct TA injection into the digital arteries, has also been reported [7]. TA injection may also cause acute retinal necrosis with photopsia, photosensitivity, and blurred vision [6]. In vitro situations have shown that exposure to TA decreases mesenchymal stromal cells in a curvilinear pattern [8]. However, contamination could have contributed to the present case, as the injection was performed in a non-sterile situation. Contaminated corticosteroid injections resulting in a multi-state fungal outbreak have been reported [6]. In the present case, the patient and his friend injected each other; his friend had no significant symptoms, which may imply that a contaminated needle or the ointment itself was the major source of inflammation.

Perineal reconstruction is challenging because the shape has a complex structure and is highly related to function [9]. In many cases, free or local flaps are required for reliable perineal coverage [10]. The pudendal thigh flap is an axial pattern flap pedicled by the superficial perineal artery and can be used bilaterally [11]. It is reliable and has a rich vas- cular supply [11]. The pudendal thigh flap preserves partial erotic sensation, potentially allowing normal sexual intercourse, and the donor site can be closed primarily $[11,12]$. In the present case, both donor sites were easily closed, undermining the side of lateral thigh at the subcutaneous plane with minor dog ear deformities.

In conclusion, foreign material injections for penile augmentation are mainly conducted by laymen in contaminated situations [1]. Education regarding the disastrous side effects is desperately needed. This rare case showed the devastating results of self TA injection in non-sterile environment and favorable reconstruction outcome with multiple modalities including penile skin grafts and bilateral local flaps which successfully cover the complex three dimensional perineal areas.

\section{References}

1. Inn FX, Imran FH, Ali MF, et al. Penile augmentation with resultant foreign material granuloma and sequalae. Malays J Med Sci 2012;19:81-3.

2. Santucci RA, Zehring RD, McClure D. Petroleum jelly lipogranuloma of the penis treated with excision and native skin coverage. Urology 2000;56:331.

3. Francis J, Poh Choo Choo A, Wansaicheong Khin-Lin G. UItrasound and MRI features of penile augmentation by "Jamaica Oil" injection. A case series. Med Ultrason 2014;16: 372-6.

4. Rollin CE, Reiber G, Guinee DG, et al. Desseminated lipogranuloma and sudden death from self administered mineral oil injection. Am J Forensic Med Pathol 1997;18:100-3.

5. Hamishehkar H, Nokhodchi A, Ghanbarzadeh S, et al. Triamcinolone Acetonide Oromucoadhesive Paste for Treatment of Aphthous Stomatitis Adv Pharm Bull 2015;5:277-82.

6. Berthelot JM, Le Goff B, Maugars Y. Side effects of corticosteroid injections: what's new? Joint Bone Spine 2013;80: 363-7.

7. Otero JC. Digital necrosis after triamcinolone acetonide injection for trigger thumb: case report. J Hand Surg Eur Vol 2016; 41:354.

8. Wyles CC, Houdek MT, Wyles SP, et al. Differential cytotoxicity of corticosteroids on human mesenchymal stem cells. Clin Orthop Relat Res 2015;473:1155-64.

9. Hashimoto I, Abe Y, Nakanishi H. The internal pudendal artery perforator flap: free-style pedicle perforator flaps for vulva, vagina, and buttock reconstruction. Plast Reconstr Surg 2014;133:924-33.

10. Hong JP, Kim CG, Suh HS, et al. Perineal reconstruction with multiple perforator flaps based on anatomical divisions. Microsurgery 2017;37:394-401. 
Na WG et al.

Penile inflammation after steroid injection

11. Monstrey S, Blondeel P, Van Landuyt K, et al. The versatility of the pudendal thigh fasciocutaneous flap used as an island flap. Plast Reconstr Surg 2001;107:719-25.
12. Hollenbeck ST, Toranto JD, Taylor BJ, et al. Perineal and Lower Extremity Reconstruction Plast Reconstr Surg 2011;128: 551e-563e. 